\title{
HIDROCARBONETOS POLICÍCLICOS AROMÁTICOS EM SEDIMENTOS SUPERFICIAIS NA BAÍA DE TODOS OS SANTOS - NORDESTE DO BRASIL.
}

\author{
SANT'ANNA Jr., Nilson ${ }^{1 *}$ \\ BERETTA, Magda ${ }^{2}$ \\ TEIXEIRA, Sonilda Maria ${ }^{3}$ \\ TAVARES, Tania Mascarenhas ${ }^{3}$
}

${ }^{1}$ Universidade Federal de Pernambuco Departamento de Oceanografia -

Universidade Federal de Pernambuco (UFPE); ${ }^{2}$ Escola Politecnica, Departamento de Engenharia Ambiental - Universidade Federal da Bahia (UFBA) - Brasil; ${ }^{3}$ Instituto de Química, Departamento de Química Analítica - Universidade Federal da Bahia (UFBA) - Brasil.

\section{RESUMO}

O objetivo deste estudo foi avaliar a contaminação de 24 HPAs em sedimentos superficiais, em zonas de infra e mesolitoral, identificar as possíveis fontes desses compostos e avaliar os impactos causados na zona costeira da Baía de Todos os Santos, Bahia - Brasil. As análises foram realizadas em um cromatógrafo a gás, acoplado a um espectrômetro de massas (CG-MS). A maior concentração do total de HPAs encontrada foi na estação M14 - Saubara ( $7221,3 \mathrm{ng} / \mathrm{g}$ massa seca), e a menor concentração foi verificada na estação M07 - Ilha de Cajaiba $(48,6 \mathrm{ng} / \mathrm{g}$ massa seca). A distribuição dos HPAs dentro da BTS é governada não só por suas fontes geradoras mais por condições oceanográficas como o fluxo das correntes existentes dentro da BTS. Os resultados encontrados pelas razões moleculares e a análise dos componentes principais (PCA) mostram que as localidades estudadas na BTS apresentam uma predominância de compostos originados através de processos de combustão (pirolíticos), no entanto, a razão Ant/178 demonstrou uma forte contribuição de HPAs de origem petrogênica, provavelmente em conseqüência das atividades de produção, transporte e refino de petróleo na BTS. Os sedimentos da BTS apresentam um grau de contaminação que vai de baixo a alto o que pode comprometer a sua qualidade toxicológica. Algumas estações apresentam concentrações de HPAs individuais acima do Probable Effect Level (PEL), sugerindo desta forma que estes sedimentos podem causar efeitos adversos em organismos.

\section{ABSTRACT}

The aim of this study was to evaluate the contamination of 24 PAHs in surface sediments in areas of medium and mesolittoral, identify possible sources of these compounds and assess the impacts on the coastal area of Baía de Todos os Santos (BTS), Bahia - Brazil. Analyses were performed on a gas chromatograph coupled to mass spectrometry (GC-MS). The highest concentration of total PAHs was found at station M14 - Saubara (7221.3 ng/g dry mass) and the lowest concentration was found at station M07 - Cajaiba Island (48.6 ng/g dry mass). The distribution of PAHs in the BTS is governed not only by generating more sources for oceanographic conditions as the flow of currents within the existing BTS. The results for the reasons molecular and principal components analysis (PCA) show that the sites studied in the BTS have a predominance of compounds generated by combustion processes (pyrolytic), however, the reason Ant/178 demonstrated a strong contribution of PAHs petrogenic origin, probably as a result of production activities, transportation and refining of oil in the BTS. The sediments of the BTS with a degree of contamination ranging from low to high which can compromise their quality toxicology. Some stations have individual PAHs concentrations above the Probable Effect Level (PEL), thus suggesting that these sediments may cause adverse effects on organisms. 
SANT'ANNA Jr. et al. Hidrocarbonetos policíclicos aromáticos em sedimentos superficiais na Baía de Todos os Santos - Nordeste do Brasil.

\section{1 - INTRODUÇÃO}

A zona costeira da Bahia é a mais extensa do litoral brasileiro destacando-se a Baía de Todos os Santos (BTS). A BTS é uma das maiores baías do Brasil com $1.086 \mathrm{~km} 2 \mathrm{de}$ extensão. Fortemente influenciada por massas d'água oceânicas, possui profundidade média em torno de 6 metros (podendo atingir profundidades de até $102 \mathrm{~m}$ ) e correntes com velocidade de $41,0 \mathrm{~cm} . \mathrm{s}^{-1}$ (Orge et al., 2006), possui uma grande importância econômica e histórica regional. É uma grande receptora da carga de compostos químicos atmosféricos e continentais resultantes das atividades industriais instaladas no seu litoral.

As águas da Baía de Todos os Santos são utilizadas para várias finalidades pela população ribeirinha que reside em torno dela. As principais atividades desenvolvidas são a pesca de subsistência e comercial no interior da baía, incluindo-se a mariscagem e a aqüicultura.

A industrialização do entorno da BTS teve início na década de $50 \mathrm{com}$ a instalação de uma refinaria de petróleo desenvolvendo atividades de exploração, refino, armazenamento e transporte de petróleo e derivados, atualmente é a região de maior concentração demográfica e industrial do Estado. Por possuir uma extensa área para navegação a BTS possui diversos portos, cais e terminais marítimos que proporcionam uma intensa atividade naval tornando-os assim importantes contribuintes de hidrocarbonetos para o ambiente (Tavares, 1988).

Os Hidrocarbonetos Policíclicos Aromáticos (HPAs) são contaminantes orgânicos persistentes e são constituídos pôr uma numerosa e diversa classe de moléculas orgânicas (Yunker et al., 2002b). Constituem uma família de compostos caracterizados por possuírem dois ou mais anéis aromáticos condensados. Estas substâncias têm ampla distribuição nos mais variados compartimentos ambientais na forma de misturas complexas. Os HPAs possuem alta persistência no ambiente, baixa biodegrabilidade e são lipofílicos, alguns deles são altamente tóxicos e são considerados multagênico/carcinogênico.

Vários processos de distribuição, transporte e transformação determinam o destino de HPAs individuais e de suas misturas. Os mais importantes em áreas costeiras são a partição entre ar e água, entre água e sedimento e entre água e biota. Outros fatores que podem interferir na distribuição de HPAs no ambiente dependem de suas propriedades físico-químicas tais como solubilidade em água, pressão de vapor, constante de Henry, coeficiente de partição octanol - água (Kow) e coeficiente de adsorção em sedimento ou partição com carbono (Koc) (IPCS, 1998).

A composição de HPAs no ambiente está diretamente relacionada com sua fonte geradora. As propriedades destes compostos no ambiente poderão ser alteradas quando fatores físicos, químicos ou biológicos atuarem seletivamente ou diferentemente em suas estruturas. Por exemplo, sedimentos superficiais podem favorecer mais facilmente a degradação bacteriana de alguns HPAs, principalmente os de baixa massa molecular. A granulometria, o tipo de sedimento, se anaeróbio ou não e quantidade de carbono orgânico presente também podem ser um fator determinante para a presença de HPAs nos sedimentos.

As fontes de HPAs para o ambiente podem ter origem biogênica ou antropogênica: As principais fontes antropogênicas são: Transformação, transporte, derramamento e principalmente queima de petróleo e seus derivados, efluentes líquidos (industriais e domésticos), incineração de lixo. A contribuição de fontes biogênicas deve ser considerada bem menor quando comparada ao montante transportado pôr fontes antropogênicas (Yunker et al., 2002a; Colombo et al., 2005; Arias et al., 2010). Os HPAs termodinamicamente gerados, como aqueles obtidos durante a lenta maturação do petróleo, são formados, em sua grande maioria, por compostos de baixa massa molecular com predominância de HPAs alquilados (Baumard et al., 1999).A introdução de 
HPAs em ambientes marinhos, principalmente os estuários, pode ocorrer por diversas vias sendo as principais o transporte atmosférico, a drenagem urbana, efluentes domésticos e industriais, derramamento e deposição de óleo e derivados de petróleo por embarcações. Uma vez dentro destes ambientes, o principal destino dos HPAs é o material em suspensão na água e/ou os sedimentos, isto em conseqüência do caráter hidrofóbico que esses compostos apresentam (Law et al. 1994).

O objetivo deste estudo é avaliar a contaminação de 24 HPAs em 18 amostras de sedimentos superficiais de ambiente marinho, nas zonas de infra e mesolitoral, identificar as possíveis fontes desses compostos e avaliar os impactos causados na zona costeira da BTS, Bahia - Brasil.

\section{2 - MATERIAL E MÉTODOS}

\section{1 - Coleta das Amostras e Extração dos HPAs}

Na BTS foram coletadas 18 amostras de sedimentos superficiais, sendo 14 estações no mesolitoral e 4 estações (M15, M16, M17 e M18) localizadas no infralitoral (Figura 1). Os sedimentos da zona de mesolitoral foram coletados a partir de 20 subamostras retiradas da camada superior do sedimento $(2-10 \mathrm{~cm})$ de uma área de cerca de $25 \mathrm{~m}^{2}$. As subamostras foram coletadas com espátula de aço inoxidável e transferidas para recipiente de alumínio previamente descontaminados. As amostras do infralitoral foram coletadas a partir de material obtido com dragas do tipo Shipek, para cada estação foram realizados três lançamentos. Cada amostra foi composta de subamostras dos primeiros 2 $\mathrm{cm}$ da camada sedimentar dragada, cuidadosamente retiradas com espátula de aço inoxidável, colocadas em recipientes de alumínio descontaminados com acetona e metanol e identificadas para posterior liofilização.

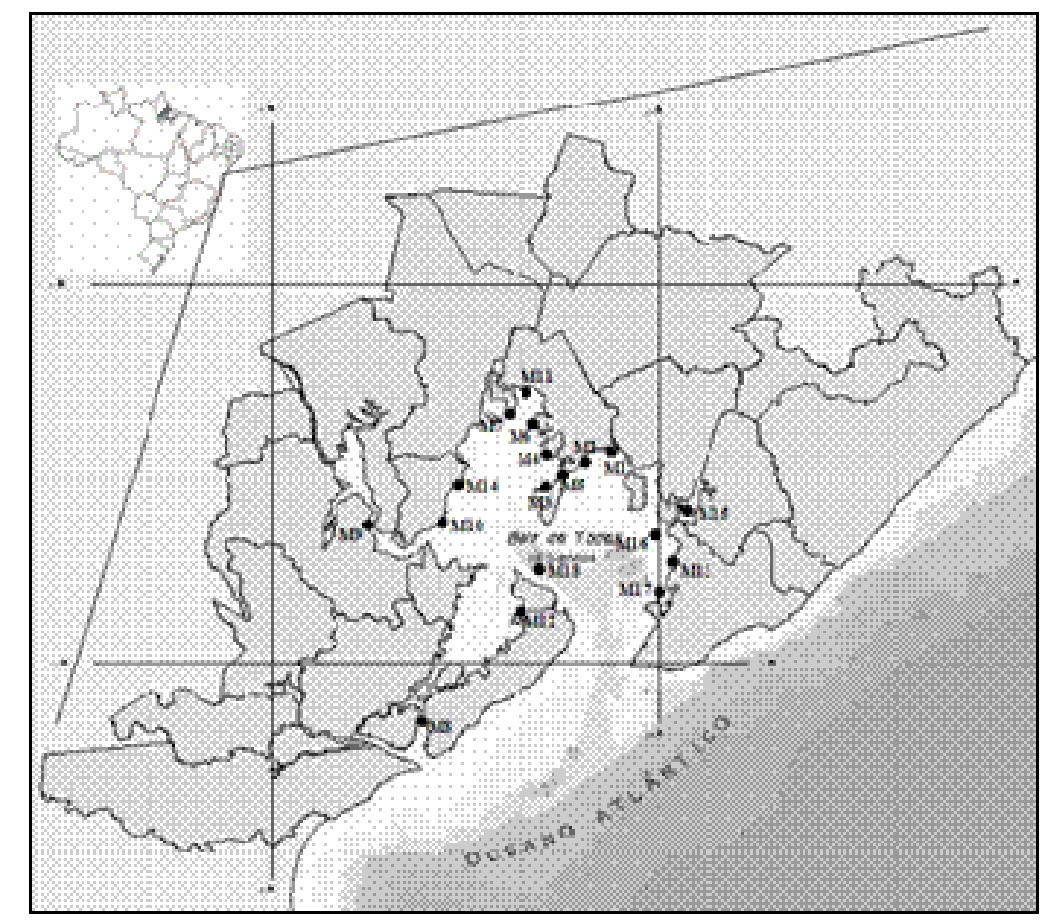

Figura 1: Área de estudo e locais de amostragem

Aproximadamente $10 \mathrm{~g}$ de sedimento liofilizado foi pesado com exatidão em tubos de vidro para centrífuga de $100 \mathrm{~mL}$. Para a extração dos HPAs foram adicionadas as amostras três porções consecutivas de $30 \mathrm{~mL}$ de diclorometano. A cada adição, com o auxílio de um bastão de vidro, a amostra era macerada com o intuito de promover uma melhor interação da mesma com o solvente. As amostras foram submetidas à sonicação em banho de ultrasom por 5 minutos e centrifugadas a 3000 rpm durante 3 minutos. 
SANT'ANNA Jr. et al. Hidrocarbonetos policíclicos aromáticos em sedimentos superficiais na Baía de Todos os Santos - Nordeste do Brasil.

Após a centrifugação, com o auxílio de pipeta Pasteur, o sobrenadante foi transferido para um balão de $250 \mathrm{~mL}$ onde foi rotaevaporado a temperatura de $39{ }^{\circ} \mathrm{C}$ até volume aproximado de $1 \mathrm{~mL}$, este extrato foi transferido para vials com capacidade de $2 \mathrm{ml}$ e secos em corrente de nitrogênio.

A purificação e o fracionamento dos extratos foram feitos por cromatografia de adsorção em colunas de vidro ( $300 \mathrm{~mm}$ de comprimento e $10 \mathrm{~mm}$ de diâmetro interno). O preenchimento da coluna foi iniciado com lã de vidro para evitar escoamento dos adsorventes. Depois foi introduzido na coluna $8 \mathrm{~g}$ de sílica ativada e desativada parcialmente submersa em n-Hexano e agitadas levemente com o auxílio de um bastão de vidro para eliminação de bolhas de ar. Em seguida o mesmo procedimento foi realizado para $8 \mathrm{~g}$ de alumina. O preenchimento da coluna foi finalizado com uma pequena porção de cobre em pó ativado em ácido clorídrico $1 \mathrm{M}$, seguida de Sulfato de Sódio.

Os extratos depois de secos em rotaevaporador, foram transferidos para o topo da coluna com o auxílio de pipeta Pasteur e o extrato foi fracionado em duas porções. A primeira fração (F1) foi eluída com $20 \mathrm{ml}$ de n-Hexano e a segunda fração (F2), contendo os HPAs, foi eluída com uma mistura de n-Hexano e Diclorometano $(40 \mathrm{ml})$ na proporção de $4: 1 \mathrm{v} / \mathrm{v}$. Após a eluição cada fração foi concentrada em rotaevaporador, nas mesmas condições da extração, até um volume aproximado de $1 \mathrm{ml}$ e transferidos para vial com capacidade de $1,8 \mathrm{ml}$. Os extratos foram secos em corrente de $\mathrm{N} 2$ de alta pureza. Os extratos secos foram conservados em freezer a $-25^{\circ} \mathrm{C}$ até o momento das análises.

\section{2 - Controle de Qualidade das Análises e Quantificação dos HPAs}

Antes do início da extração, foram adicionados diretamente nas amostras padrões deuterados (Fenantreno D10 e Benzo(a)Antraceno D12 na concentração de $1 \square \mathrm{g} / \mathrm{ml}$ ), para verificação da eficiência do método. A recuperacão do Fenantreno D10 ficou em $62,5 \pm 15,8 \%$ e a do Benzo(a)Antraceno D12 em 77,1 $\pm 11,1 \%$.

O controle de qualidade das análises de HPAs em sedimentos também foi realizado com material certificado fornecido pela National Institute of Standards and Technology (NIST - 1944 WaterWay Sediment). A análise foi realizada em triplicata, o composto que apresentou a melhor recuperação foi o Fluoranteno $(100 \% ; 8938 \pm 1372)$ e o pior foi o 1 Metilfenantreno (53\%; 900,0 $\pm 81,70)$.

Os HPAs foram identificados utilizando-se o padrão certificado SRM - 1491 contendo 24 HPAs [Naftaleno (Naf); 2-Metilnaftaleno (2MetNaf); 1-Metilnaftaleno (1MetNaf); Bifenil (Bif); 2,6-Dimetilnaftaleno (DiMetNaf); Acenaftileno (Aceftl); Acenafteno (Acef); 2,3,5-Trimetilnaftal (TriMetNaf); Fluoreno (Flu); Fenantreno (Fen); Antraceno (Ant); 1-Metilfenantreno (MetFen); Fluoranteno (Fluor); Pireno (Pir); Benzo(a)Antraceno (BaAnt); Criseno (Cri); Benzo(b+K)Fluoranteno (BbkFlu); Benzo(e)Pireno (BePir); Benzo(a)Pireno (BaPir); Perileno (Per); Indeno + Dibenzo (ID); Benzo(ghi)Perileno (BghiPer)] adquiridos da NIST.

Soluções padrões contendo os 24 compostos de interesse, variando nas concentrações de 0,5 a 2,0 ng/g foram injetadas no aparelho, nas mesmas condições que as amostras. A partir dos resultados das soluções padrões foram feitas curvas de calibração para cada um dos 24 compostos analisados. Para a quantificação dos compostos foram utilizados dois padrões internos, HPAs deuterados, sendo eles: Naftaleno D8 e Pireno D10. Aos extratos secos, momentos antes das análises, foram adicionados $200 \mu \mathrm{L}$ de Isooctano e $100 \mu \mathrm{L}$ do padrão interno perfazendo um volume final de diluição de $300 \mu \mathrm{L}$ da amostra. Os compostos identificados nas análises foram quantificados através da relação de suas áreas com as do padrão interno. As análises dos HPAs foram realizadas em um cromatógrafo a gás, modelo $3600 \mathrm{cx}$ da Varian, acoplado a um espectrômetro de massas do tipo Ion-trap, também da Varian (Modelo 2000), com detector MS/MS e amostrador automático (Auto-sampler). A identificação dos compostos se deu pelo sistema de aquisição no modo de sistema de monitoramento de íons (SIM). A 
coluna capilar utilizada para a separação dos compostos foi uma Factor Four, DB - 5 $(30 \mathrm{~m} \times 0,25 \mathrm{~mm} \times 0,25 \mu \mathrm{m})$. Foram injetados $2 \mu \mathrm{L}$ de cada amostra, o gás de arraste utilizado foi o hélio (He) com vazão de $1,1 \mathrm{~mL} \cdot \mathrm{min}^{-1} \mathrm{e}$ as injeções feitas no modo splitless. A programação da temperatura da coluna foi iniciada de $60^{\circ} \mathrm{C} / \mathrm{min}$ e $120^{\circ} \mathrm{C}$ numa taxa de $15^{\circ} \mathrm{C} / \mathrm{min}$ e de 120 a $320^{\circ} \mathrm{C}$ numa taxa de $10^{\circ} \mathrm{C} / \mathrm{min}$, permanecendo nesta temperatura por 4 minutos. As amostras também foram submetidas à análise granulométrica para determinação das Frações Areia (Grossa e Fina), Silt e Argila e $C_{\text {org }}$.

\section{3 - RESULTADOS E DISCUSSÃO}

\section{1 - Concentração de HPAs nas amostras de sedimento}

A maior concentração do total de HPAs foi encontrada na estação M14 - Saubara (7221,3 ng/g massa seca), área altamente urbanizada, por se tratar de área turística apresenta intenso tráfico de embarcações de passeio e de pesca. A menor concentração de HPAs foi verificada na estação M07 - Ilha de Cajaiba (48,6 ng/g massa seca) (tabela 1). Outras estações que apresentaram concentrações elevadas (M12 - Baiacu; M13 Lobato; M15 - Base Naval 1; M16 - Base Naval 3; M17 - Porto da Ribeira) contêm uma maior proporção de compostos de alta massa molecular (4 - 6 anéis), do Fluoranteno ao Benzo(ghi)Perileno. Estas localidades também apresentam intenso tráfico de embarcações de pequeno, médio e grande porte. Os altos valores nas estações M15 e M16 também podem estar sendo favorecidas pela consistência de seus sedimentos, que apresentaram valores mais elevados para argila. Sedimentos argilosos tendem a reter com mais facilidade HPAs.

A estação M15 está localizada dentro de outra baía, chamada de Baía de Aratú. Local de intenso tráfego marítimo é um dos mais importantes centros de manutenção industrial da marinha do Brasil. A Baixa circulação de águas dentro desta baía e a contribuição das águas do rio Cotegipe proporcionam condições favoráveis para as altas concentrações de HPAs verificadas nesta estação. As altas concentrações também encontradas na estação M16, saída da baia de Aratú, indica que estes compostos estão sendo exportados para toda a BTS.

O composto que apresentou a menor contribuição para o $\square$ HPAs, em todas as amostras analisadas, foi o Acenaftileno. O predomínio de HPAs de maior massa molecular deve-se provavelmente às suas altas persistências no ambiente, consequência do elevado caráter lipofílico e hidrofóbico destes compostos. As maiores concentrações do $\square$ HPAs de alta massa molecular variou entre 1060,5 ng/g (M16 - Base Naval 3) e 6232,9 $\mathrm{ng} / \mathrm{g}$ (M14 - Saubara) (Figura 2), estas localidades apresentam uma intensa aitividade de embarcações, de pequeno e grande porte, para atividades militares, industriais e/ou de pesca.

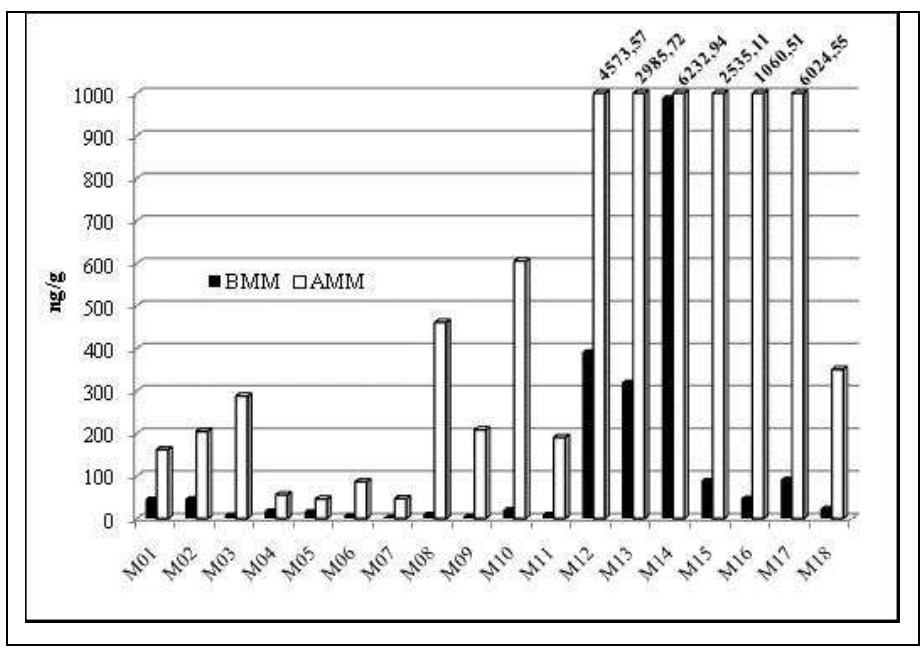

Figura 2: Distribuição do $\square$ HPAs de alta e baixa massa molecular encontrados nas amostras de sedimentos superfiais da Baía de Todos os Santos. 
SANT'ANNA Jr. et al. Hidrocarbonetos policíclicos aromáticos em sedimentos superficiais na Baía de Todos os Santos - Nordeste do Brasil.

A distribuição dos HPAs dentro da BTS é governada não só por suas fontes geradoras mais por condições oceanográficas como o fluxo das correntes existentes dentro da BTS. Deve-se considerar que o fluxo de águas marinhas é muito superior ao de água doce. Segundo Lessa et al., 2001 as correntes na baía de todos os santos são predominantemente bidirecionais, com destaque para correntes circulares que ocorrem no centro da baía. As correntes costeiras são induzidas principalmente pelas correntes mareais e correntes provocadas pelos ventos, responsáveis pela circulação das águas mais superficiais.

As concentrações do $\square$ HPAs encontrados nos sedimentos superficiais deste estudo, quando comparados com uma escala de contaminação sugerida por Benlahcen et al., 1997 (Altamente contaminado > 3000; Moderadamente contaminado entre 200 - 3000; baixa contaminação $<200 \mathrm{ng} / \mathrm{g}$ ) indicam que os sedimentos coletados na BTS apresentam um grau de contaminação que vai de baixa contaminação a altamente contaminado (Tabela 2).

Não foram observadas correlações significativas entre as concentrações de HPAs com as concentrações de Carbono orgânico encontradas nos sedimentos analisados. Está registrado na literatura (Ke et al., 2005; Yunker et al., 2002a; Yunker et al., 2002b; Viguri et al.,2002) que as características dos sedimentos influenciam na distribuição e concentração dos HPAs. Importantes fatores incluem composição química da matéria orgânica e a presença da fração mais fina do sedimento. Neste trabalho também não foram encontradas correlações significativas entre as concentrações do $\square$ HPAs e as percentagens da fração mais fina do sedimento estudado (Silt e Argila). Este fato sugere que a presença de HPAs não está dependendo exclusivamente das propriedades dos sedimentos, o que é um forte indicador de que as reações controladoras das concentrações de HPAs na BTS podem estar fora deste ambiente, ou que as proximidades das fontes geradoras de HPAs podem estar mascarando processos ambientais normalmente considerados controladores destas concentrações.

\section{2 - Fontes de HPAs para a BTS}

O método para identificação das fontes de HPAs mais apropriado e amplamente divulgado na literatura internacional (Law et al., 1994; Budzinsk et al., 1997; Baumard et al., 1998a; Baumard et al., 1998b; Baumard et al.,1999; Stella et al., 2002; De Luca et al., 2005; Walker et al., 2005) baseia-se na razão entre um HPA e seu isômero para a identificação das fontes de HPAs.

HPAs de BMM (HPAs com a razão $m / z<202$ ) são os principais constituintes do petróleo enquanto que HPAs de AMM (HPAs com a razão $m / z \square$ 202) tem suas origens associadas a fontes pirolíticas. Resultados da razão $\square \mathrm{AMM} / \square \mathrm{BMM}>1$ indicam origem pirolítica assim como resultados para razões $\square$ AMM/ $\square \mathrm{BMM}<1$ indicam origem petrogênica. Os resultados encontrados nas amostras para a razão $\square$ AMM / $\square$ BMM indicam uma predominância de HPAs de origem pirolítica, apesar disto não se deve desconsiderar a contribuição de HPAs de origem petrogênica. A presença de HPAs de baixo peso molecular e HPAs alquilados, nos resultados encontrados, vêm fortalecer esta possibilidade.

Para uma melhor elucidação das origens dos HPAs encontrados nos sedimentos superficiais da BTS e do litoral Norte da Bahia foram utilizados os seguintes índices: Ant / Ant + Fen - (Ant/178); B(a)Ant / B(a)Ant + Cris - (B(a)A/228); Fluor / Fluor + Pir, sugeridas por Yunker et al. (2002a) (Tabela 3). Os resultados destas razões mostram uma predominância de compostos originados através de processos de combustão, com exceção para a razão Ant/178 que também demonstrou uma forte contribuição de HPAs de origem petrogênica.

HPAs com massa molecular 178 a 202 são comumente usados para distinguir fontes de HPAs entre pirolíticas e petrogênica. Quando a razão Ant / Ant + Fen for < 0.10 (Estações M3, M4, M8, M9, M11e M18) apresenta a uma indicação de fonte 
petrogênica, quando for $>0.10$ indica que os HPAs tem sua origem através de processos de combustão. Para Fluor/Fluor + Pir a razão abaixo de 0,40 mostra um indicativo de HPAs de origem petrogênica (Estações M2, M7 e M8), quando for maior que 0,40 indicam HPAs com origem na emissão de carros e caminhões através da combustão de, gasolina, diesel ou querosene, podem ter também sua origem na queima de madeira ou de biomassa. HPAs com alta massa molecular (entre 228 e 276) são os menores constituintes para produtos refinados do petróleo e estão presentes em quantidades significantes em asfalto, betume e carvão. Quando as razões B(a)Ant/228 for menor que 0.20 indica HPAs com origem petrogênica (M1) e quando maior que 0.20 HPAs de origem pirolítica (Budzinski et al., 1997, Yunker et al., 2002a).

Apesar das razões acima expostas já fornecerem uma indicação de que os sedimentos analisados neste estudo sofrem uma contaminação por HPAs de origem pirolítica, foi realizado uma outra avaliação entre as razões Fenantreno/Antraceno (Fen/Ant) e Fluoranteno/Pireno (Flu/Pir). Esta razão tem sido mais explorada por alguns autores (Budzinski et al., 1997; Benlachen et al., 1997; Baumard et al. 1998a; Soclo et al., 2000; De Luca et al., 2005). A utilização destas razões para a identificação da origem de HPAs para os sedimentos está fundamentada no fato que o petróleo, normalmente, contém mais Fenantreno do que Antraceno já que o Fenantreno é mais estável termodinamicamente. Em conseqüência disto, quando ocorrem valores > 10 da razão Fen/Ant isto indica poluição de origem petrogênica, e quando ocorrem valores < 10 isto indica poluição de origem pirolítica. Quanto à razão Flu/Pir, como o Fluoranteno é termodinamicamente menos estável do que o Pireno, este composto vai predominar nas amostras caracterizando desta forma HPAs de origem pirolítica, quando ocorrer o inverso a origem dos HPAs é petrogênica. Segundo Baumard et al., 1999 a contaminação é considerada de origem pirolítica quando a razão Flu/Pir é maior que 1 , e de origem petrogênica quando ela é menor que 1. Na figura 3 encontra-se um gráfico da correlação entre as razões Fen/Ant e Flu/Pir para as estações estudadas neste trabalho. Esta figura auxilia numa melhor visualização das possíveis fontes de HPAs para os sedimentos analisados.

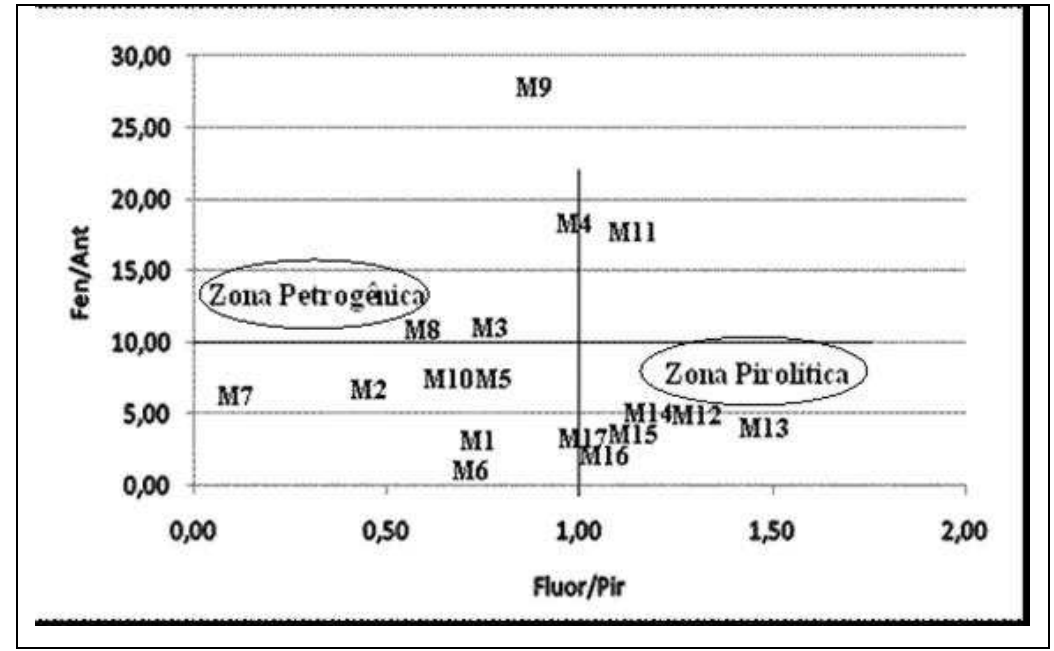

Figura 3: Correlação entre as razões de Fenantreno/Antraceno (Fen/Ant) e Fluoranteno/Pireno (Fluor/Pir)

Observa-se no referido gráfico que duas zonas foram definidas: uma é característica da origem petrogênica e a outra, pirolítica. Como pode ser visto, das 18 estações em que foi calculada as razões, boa parte pertence a zona de influência pirolítica (M12, M13, M14, M15, M16, M17), isto é, apresentaram valores da razão Fen/Aant abaixo de 10 e valores da razão Fluor/Pir acima de 1 o que é característico de processos de combustão em alta temperatura. Estas estações estão localizadas em áreas de intenso tráfego de embarcações e com forte influência da drenagem urbana. Apenas três estações (M3, M4, M8, M9) pertencem a zona de influência petrogênica. As estações M3 e M4 estão localizadas próximas a refinaria de petróleo e porto responsável pelos 
SANT'ANNA Jr. et al. Hidrocarbonetos policíclicos aromáticos em sedimentos superficiais na Baía de Todos os Santos - Nordeste do Brasil.

produtos manufaturados nesta refinaria, respectivamente. As demais estações apresentaram resultados conflitantes, sugerindo um leve impacto petrogênico sobre uma predominante origem pirogênica. Apesar dos resultados sugerirem uma forte influência pirolítica na composição de HPAs na BTS, não se pode concluir que os HPAs de alta massa molecular tenham uma origem diferenciada daqueles de baixa massa molecular, pois fatores como as taxas de degradação podem influenciar nas concentrações destes compostos nos sedimentos superficiais da BTS.

Ambientes marinhos, especialmente os estuarinos, por serem extremamente dinâmicos, podem apresentar condições únicas para a especiação de HPAs e assim mascarar a identificação de suas fontes. A dinâmica natural dos sistemas estuarinos, a resuspensão das camadas de sedimentos durante a movimentação das marés e a homogeneização destas camadas de sedimento pode dificultar a identificação e especificação da fonte geradora destes poluentes (Rogers, 2002).

Deve - se considerar que as zonas estuarinas e costeiras não estão apenas sujeitas a impactos sofridos pelo lançamento direto de poluentes, outras vias também podem servir como contribuinte de poluição para estas áreas, uma dessas vias pode ser a atmosférica. De acordo com Rocha et al., 2009; Guarieiro et al., 2009 estudando os 16 HPAs prioritários definidos pela EPA n\a região das estações M15 e M16 determinaram que em média, $\Sigma$ PAH representou $0,005 \%$ da massa do material particulado atmosférico nesta região e de 0,010\% para a região da estação M8.

Outro teste para verificação das fontes de HPAs para a BTS fundamenta - se na correlação entre o $\square$ HPAs e a concentração do Benzo(a)Pireno. Esta correlação foi realizada baseando-se no fato da concentração do Benzo(a)Pireno ser desprezível no petróleo, portanto concentrações elevadas no ambiente indicam que este composto possuem origem pirolítica.

Este trabalho apresentou uma boa correlação $(r=0,77)$ entre as concentrações do Benzo(a)Pireno e o $\square$ HPAs para os resultados encontrados, reforçando assim a dominância de HPAs de origem pirolítica no ambiente. Na figura 4 encontra-se a correlação entre o Benzo(a)Pireno e o $\square$ HPAs.

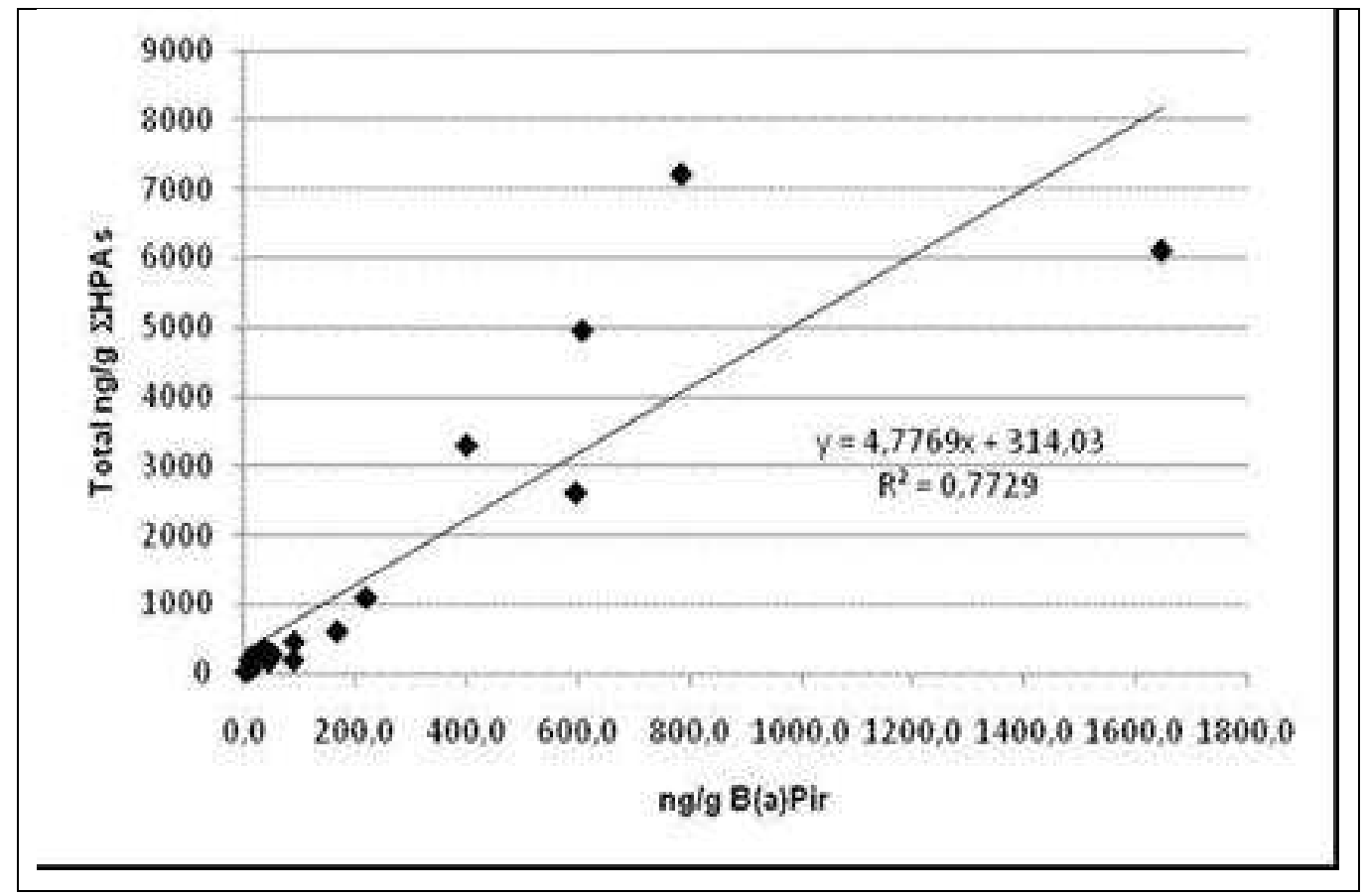

Figura 4: Correlação entre as concentrações do $\square$ HPAs X Benzo(a)Pireno nos sedimentos superficiais da BTS. 


\section{3 - Análise dos Componentes Principais}

Para a análise dos componentes principais, foi formada uma matriz com os 22 HPAs analisados e as estações de estudo, tanto para sedimentos como para moluscos. Esta matriz foi submetida a uma padronização por fileiras e em seguida, foi calculada a similaridade por correlação momento-produto de Pearson e computados os valores da matriz de dispersão, estando associada a cada um desses autovalores e autovetores, que corresponderam aos eixos principais do espaço multidimensional.

O resultado da análise dos componentes principais mostra um percentual cumulativo para os três vetores de $97,1 \%$. No vetor 1 , representado pelo grupo I, que explica $58,9 \%$ dos dados, mostra que a maioria das estações possuem uma associação direta com compostos de alta massa molecular reforçando uma contribuição pirolítica. No entanto, não deve-se descartar uma contribuição petrogênica já que alguns compostos de baixa massa molecular também apresentam uma associação direta com estas estações. Deve-se destacar também que estas estações foram as que apresentaram as concentrações de $\square$ HPAs mais elevadas. 0 vetor 2 , representado pelo grupo II, que explica $29,5 \%$ dos dados está formado exclusivamente por compostos de baixa massa molecular indicando desta forma que estes composto apresentam uma associação inversa provavelmente em decorrência das suas baixas concentrações quando comparados com as concentrações mais elevadas encontradas para compostos de alta massa molecular. O vetor 3, representado pelo grupo III, que explica $8,7 \%$ dos dados, não apresentam nenhuma associação entre as estações de infralitoral e as estações M12, M13 e M14. Este grupo de estações foram as que apresentaram os maiores níveis de contaminação e no qual a presença de todos os compostos analisados foi significativa indicando que a contaminação pode ser tanto pirolítica quanto petrogênica. As características ambientais associadas a cada estação também influenciam na distribuição dos HPAs em seus compartimentos ambientais, sobretudo o sedimento que na maioria das vezes torna-se reservatório final para estes compostos (Figura 5).

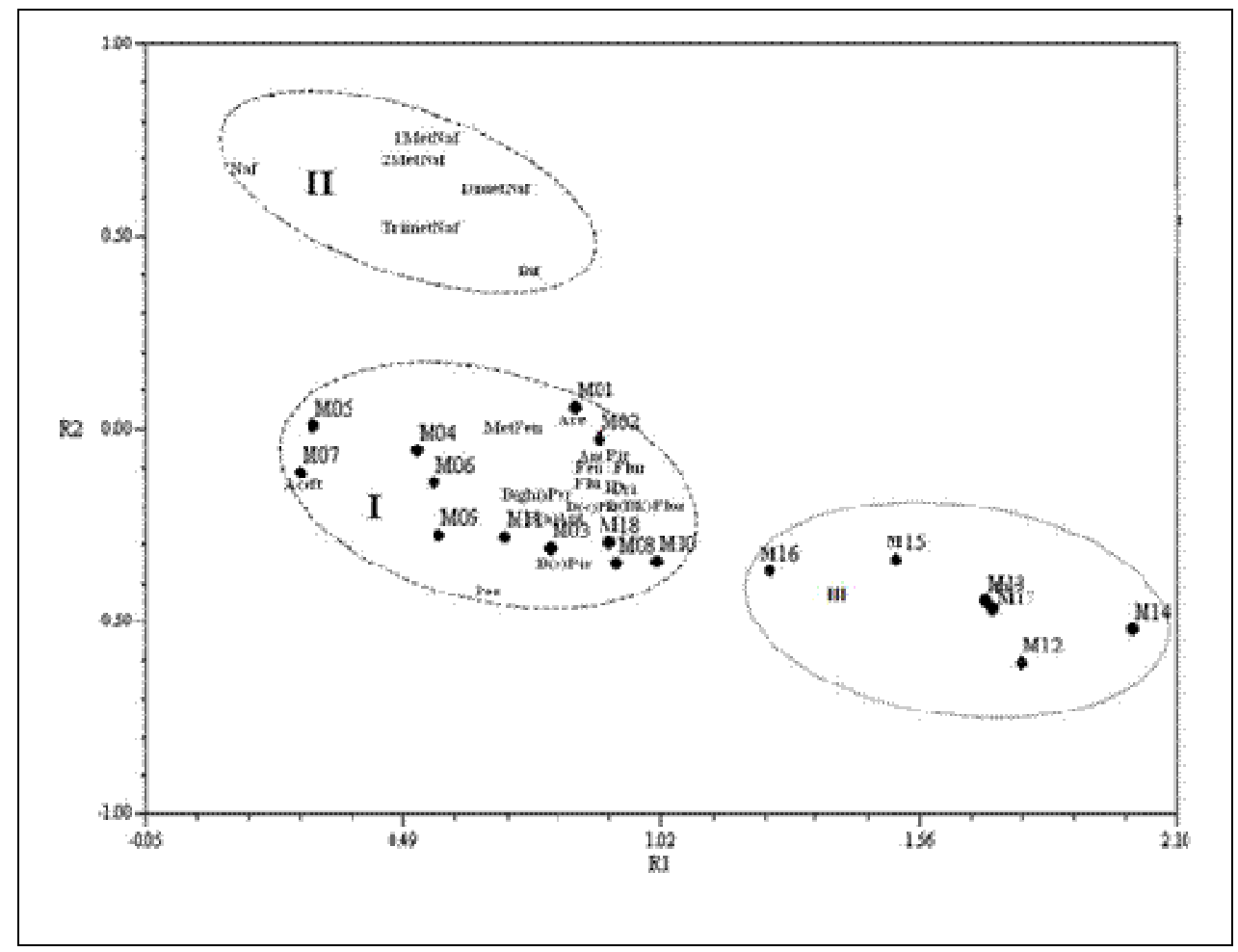

Figura 5: Análise dos componentes principais para as amostras de sedimentos superficiais coletados na BTS.

\section{4 - Qualidade dos Sedimentos Superficiais da BTS}


SANT'ANNA Jr. et al. Hidrocarbonetos policíclicos aromáticos em sedimentos superficiais na Baía de Todos os Santos - Nordeste do Brasil.

Os critérios de qualidade de sedimentos estabelecidos pela agência ambiental do Canadá e os implantados pela National Oceanic and Atmospheric Administration (NOAA) dos Estados Unidos são os mais utilizados por pesquisadores do mundo (Baumard et al., 1999; Yunker et al., 2002b; Xu et al., 2007).

Os critérios da agência ambiental do Canadá dividem-se em dois níveis (Environmental Canadá, 1998; Lee et al., 1999): o mais baixo, denominado TEL (Threshold Effect Level) e o mais alto, denominado PEL (Probable Effect Level). Estes dois níveis delimitam intervalos de probabilidade de ocorrência de efeitos biológicos adversos. Abaixo do menor nível espera-se que raramente seja observado algum efeito adverso e acima do maior nível espera-se observar algum efeito adverso com maior freqüência. Valores entre os dois níveis pode apresentar algum efeito adverso. Estes critérios foram utilizados neste trabalho para avaliar a qualidade dos sedimentos analisados (Tabela 4). Os critérios determinados por esta agência são utilizados prioritariamente como ferramentas de interpretação flexíveis para a avaliação da significância toxicológica ambiental dos dados químicos do sedimento proporcionando desta maneira, a tomada de ações e decisões para o gerenciamento de ambientes impactados.

Não Carcinogênico; ** Carcinogênico; *** Fortemente Carcinogênico Das 19 estações estudadas na BTS a M14 - Saubara foi a que apresentou concentrações que podem provocar efeitos adversos nos organismos para os compostos Fenantreno, Criseno e Benzo(a)Pireno. Esta estação foi a que apresentou as maiores concentrações de HPAs na BTS e a origem destes HPAs prioritariamente é pirolítica. A estação M17 - Porto da Ribeira também apresentou concentrações acima do PEL para o B(a)Pireno. A intensa movimentação de embarcações na área pode ser a causa principal da presença de HPAs tanto de alta como de baixa massa molecular. As figuras 6 abc mostram uma comparação entre as concentrações de Fenantreno, Criseno e B(a)Pireno encontradas nos sedimentos superficiais e entre os critérios de qualidade de sedimento definidos pela agência ambiental do Canada (TEL e PEL). 


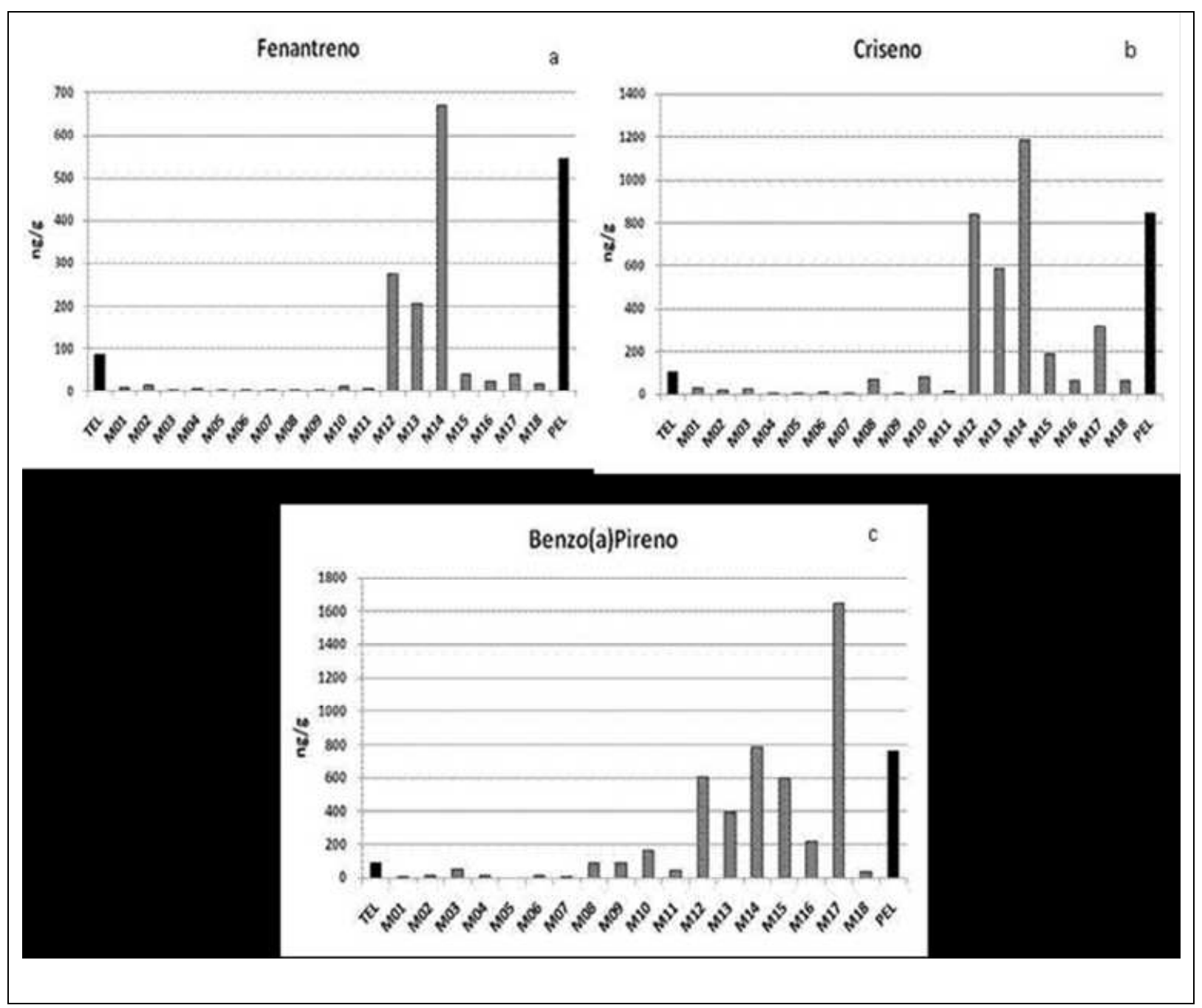

Figura 6: Comparação entre as concentrações de Fenantreno (a), Criseno (b) e Benzo(a)Pireno (c) encontradas nos sedimentos superficiais das estações estudadas e os valores de Threshold Effect Level (TEL) e Probable Effect Level (PEL).

Apesar das outras estações apresentarem concentrações abaixo do limite que possa provocar efeitos adversos em organismos deve-se levar em consideração que os sedimentos das estações localizadas na BTS apresentam um nível de contaminação que sugere que prováveis efeitos adversos podem ocorrer na biota presente nestes sedimentos já que algumas estações apresentam concentrações de HPAs individuais entre o TEL e o PEL. Deve-se ter uma atenção especial para as estações M12, M13, M14, M15, M16, M17, já que estas apresentaram as maiores concentrações de HPAs.

Segundo Bahia (2004) a sinergia das ondas com as correntes existentes na BTS pode resultar em uma elevada capacidade de transporte de sedimentos. Nesta ação conjunta as ondas exercem tensões de atrito que mobilizam e freqüentemente ressuspendem os sedimentos, que então são facilmente transportados pelas correntes. De acordo com Lessa et al., 2001, na maior parte da baía, parece prevalecer condições dominantes de vazante e a quantidade de sedimento grosso deixado atualmente na baía e insignificante, demonstrando que a presença de deltas mareais parece resultar da obstrução de transporte de sedimento costeiro por correntes saindo da baía. Medidas de correntes costeiras próximas à baía indicam que a combinação de correntes mareais com uma corrente na direção Sudoeste induzida por vento, gera fluxos orientados para Oeste capazes de transportar sedimentos para fora da boca da baía. 
SANT'ANNA Jr. et al. Hidrocarbonetos policíclicos aromáticos em sedimentos superficiais na Baía de Todos os Santos - Nordeste do Brasil.

\section{4 - CONCLUSÃO}

Os resultados verificados neste trabalho indicam que os HPAs encontrados nos sedimentos superficiais coletados na BTS são prioritariamente de origem pirolítica sugerindo uma forte influência de processos industriais e urbanos para a área de estudo. A presença de HPAs de Baixa Massa Molecular e metilados também sugere que atividades de produção, transporte e refino de petróleo contribuem para a contaminação do ambiente, o resultado verificado na análise dos componentes principais vem reforçar esta tendência. Apesar das regiões entre marés serem mais vulneráveis ao aporte de contaminantes oriundos do continente os resultados encontrados em sedimentos de infralitoral (estações M15, M16, M17 e M18) apresentam características de contaminação semelhantes aos verificados no mesolitoral indicando que o movimento das marés exerce grande influência na distribuição de HPAs na BTS.

De acordo com critérios estabelecidos pela literatura científica internacional os sedimentos da BTS apresentam um grau de contaminação que vai de baixo a alto o que pode comprometer a sua qualidade toxicológica. Isto pode ser percebido nos sedimentos analisados de algumas estações (M12, M14, e M17) onde apresentam concentrações de HPAs individuais acima do PEL, sugerindo desta forma que estes sedimentos podem causar efeitos adversos nos organismos que dependam deste substrato para o seu desenvolvimento ou sua sobrevivência.

\section{5 - REFERÊNCIAS BIBLIOGRÁFICAS}

ARIAS, A. H. VAZQUEZ-BOTELLO, A. TOMBESI, N. PONCE-VÉLEZ, G. FREIJE, H. MARCOVECCHIO, J. (2010). Presence, distribution, and origins of polycyclic aromatic hydrocarbons (PAHs) in sediments from Bahía Blanca estuary, Argentina. Environ Monit Assess 160:301-314

BAHIA - Secretaria de Desenvolvimento Urbano - SEDUR. Secretaria de Meio Ambiente e Recursos Hídricos - SEMARH. Centro de Recursos Ambientais - CRA. (2004). Diagnóstico do Grau de contaminação da Baía de Todos os Santos por metais pesados e hidrocarbonetos de petróleo a partir da análise das suas concentrações nos sedimentos de fundo e na biota associada: caracterização geral da Baía de Todos os Santos.

Salvador: Consórcio BTS Hydros CH2M Hill. 366p. I. Ilus.

BAUMARD, P.; BUDZINSKI, H.; MICHON, Q.; GARRIGUES, P.; BURGEOT, T.; BELLOCQ, J. (1998a). Origin and Bioavailability of PAHs in the Mediterranean Sea from Mussel and Sediment Records. Estuarine Coastal and Shelf Science. 47, 77 - 90.

BAUMARD, P. BUDZINSKI, H. GARRIGUES, P. (1998b). PAHs in Aracachon Bay, France: Origin and Biomonitoring with Caged Organisms. Marine Pollution Bulletin, Vol. 36, No $8,577-586$.

BAUMARD, P. BUDZINSKI, H. GARRIGUES, P. DIZER, H. HANSEN, P. D. (1999). Polycyclic aromatic hydrocarbons in recent sediments and mussels (Mytilus edulis) from the Western Baltic Sea: occurrence, bioavailability and seasonal variations. Marine Environmental Research, 47, 17 - 47.

BENLAHCEN, K. T., CHAOUI, A., BUDZINSKI, H., BELLOCQ, J., GARRIGUES, P.H. (1997). Distribution and Sources of Polycyclic Aromatic Hydrocarbons in some Mediterranean Coastal Sediments. Marine Pollution Bulletin. Vol. 34, N $\square$ 5, pp. 298 - 305.

BERNANRD, D.; PASCALINE, H.; JEREMIE, J - J. (1996). Distribuition and origin of hydrocarbon in sediments from lagoons with fringing mangrove communities - Marine Pollution Bulletin 32 (10) 734 - 739.

BUDZINSKI, H. JONES, I. BELLOCQ, J. PIÉRARD, C. GARRIGUS, P. (1997). Evaluation of Sediment Contamination by Polycyclic Aromatic Hydrocarbons in the Gironde Estuary.

Marine Chemistri, 58, 85 - 97. 
COLOMBO, J.C., BARREDA, C., BILOS, N.C., MIGOYA, M.C. AND SKORUPKA, C. (2005). Oil spill in the Rio de la Plata estuary, Argentina: 2-hydrocarbon disappearance rates in sediments and soils. Environmental Pollution, 134, 267-276.

ENVIRONMENT CANADA. (1998). Canadian Sediment Quality Guidelines for the Protection of Aquatic Life. www.ec.gc.ca/ceqg - rcqe/sediment.htm.

GUARIEIRO, A. L. N.; ROCHA DA, G. O.; CONCEIÇÃO, L. DOS S.; LOPES, W. A.; PEREIRA, P. A. DE P.; DE ANDRADE, J. B. (2009) Avaliação de HPAS em aerossóis atmosféricos da Ilha de Itaparica - BA, AB-035. In: Reunião Anual da Sociedade Brasileira de Química, 32, Fortaleza, CE, 30/05 a 02/06/2009.

IPCS - INTERNATIONAL PROGRAMME ON CHEMICAL SAFETY. Environmental Criteria 202. Selected non - heterocyclic PAHs. World Health Organization, Geneva, 1998.

KE, L.; YU, K. S. H.; WONG, Y. S.; TAM, N. F. Y. (2005). Spatial and Vertical Distribution of Polycyclic Aromatic Hydrocarbons in Mangrove Sediments. Science of the Total Environmental. 340: 177 - 187.

LAW, R. J.; BISCAYA, J. L. (1994). Polyciclic Aromatic Hydrocarbons (PAH) - Problems and Progress in Sampling, Analysis and Interpretation. Marine Pollution Bulletin, N $\square$ 4, Vol. $29-5,235-241$ pp.

LEE, B.; YI, J. (1999). A statistical approach for determining the environmental impact of polynuclear aromatic hydrocarbons in an oil spill - contaminated coastal area.

Environmental Pollution, 105, No 3, 391 - 396.

LESSA, G. C.; DOMINGUEZ, J. M. L.; BITTENCOURT, A. C. S. P. E BRICHTA, A. (2001) The tides and tidal circulation of Todos os Santos Bay, Northeast Brazil:a general characterization., Anais da Academia Brasileira de Ciências, 73(2):245 - 261.

MACIAS-ZAMORA JV, MENDOZA-VEGA E, VILLAESUSA-CELAYA JA. (2002). PAHs Composition of Surface Marine Sediments: a Comparison to Potential Local Sources in Todos Santos Bay, BC, Mexico. Chemosphere; 46:459- 68.

MASKAOUI, K., ZHOU, J.L., HONG, H.S., ZHANG, Z.L. (2002) Contamination of polycyclic aromatic hydrocarbons in the Jiulong river estuary and western Xiamen Sea, China.

Environ. Poll., 118: 109-122

NISHIGIMA, F. N., WEBER, R. R., BÍCEGO, M. C. (2001). Aliphatic and Aromatic Hydrocarbons in Sediments of Santos and Cananéia, SP, Brazil. Marine Pollution Bulletin. N $\square$ 11, Vol. 42, $1064-1072$.

NOTAR, M.; LESKOVSEK, H.; FAGANELI, J. (2001). Composition, Distribuition and Sources of Polycyclic Aromatic Hydrocarbons in Sediments of the Gulf of Trieste, Northern Adriatic Sea. Marine Pollution Bulletin, N $\square$ 1, Vol. 42, 36 - 44 pp.

ORGE, M.D.R.; PORSCHÉ, I.J.; COSTA, M.C.; LIMA, J.S.; SOARES, S.E.D.; JUSTINO, R. (2006). Assessment of oil refinery waste on Rhizophora mangle $L$. seedling growth in mangrove of Todos os Santos Bay, Bahia, Brazil. Rev. Esc. Minas vol.59 no.3: 471 477.

OU, S.M., ZHENG, J.H, ZHENG, J.S., RICHARDSON, B.J., LAM, P.K.S. (2004). Petroleum hydrocarbons and polycyclic aromatic hydrocarbons in the surficial sediments of Xiamen Harbour and Yuan Dan Lake, China. Chemosphere 56, 107-112.

ROCHA, G. O.; LOPES, W. A.; PEREIRA, P. A. P.; VASCONCELlOS, P. C.; OliVEIRA, F. S.; CARVALHO, L. S.; CONCEIÇÃO, L. S.; ANDRADE, J. B. Quantification and Source Identification of Atmospheric Particulate Polycyclic Aromatic Hydrocarbons and their Dry Deposition Fluxes at Three Sites in Salvador Basin, Brazil, Impacted by Mobile and Stationary Sources. J. Braz. Chem. Soc., Vol. 20, Nº. 4, 680-692, 2009.

SOCLO, H. H.; GARRIGUES, PH.; EWALD, M. (2000). Origin of Polycyclic Aromatic Hydrocarbons (PAHs) in Coastal Marine Sediments: Case Studies in Cotonou (Benin) and Aquitaine (France) Areas. Marine Pollution Bulletin. 40, 387 - 396. 
SANT'ANNA Jr. et al. Hidrocarbonetos policíclicos aromáticos em sedimentos superficiais na Baía de Todos os Santos - Nordeste do Brasil.

TAM, N.F.Y.; KE, L.; XANG, X.H.; WONG, Y.S. (2001) - Contamination of PAH insurface sediments of mangroves swamps - Environmental Pollution 114, 255 - 263.

TAVARES, T. M.; ROCHA, V. C.; PORTE, C.; BARCELÓ, D. AlBAIGÉS, J. (1988). Aplication of Mussel Watch Concept in studies of Hydrocarbons, PCBs and DDT in the Brasilian Bay of Todos os Santos (Bahia). Marine Pollution Bulletin, Vol. 19, N $\square 11,575$ - 578.

XU, J.; YU, Y.; WANG, P.; GUO, W.; DAI, S.; SUN, H. (2007). Polycyclic Aromatic Hydrocarbons in the Surface Sediments from Yellow River, China. Chemosphere. 2006.10.074.

YUNKER, M. B.; BACKUS, S. M., PANNATIER, E. G. JEFFRIES, D. S.; MACDONALD, R. W. (2002a). Sources and Significance of Alkane and PAH hydrocarbons in Canadian Artic Rivers. Estuarine, Coastal and Shelf Science. 55, 1 - 31.

YUNKER, M. B.; MACDONALD, W.; VINGARZAN, R.; MITCHELL, R. H.; GOYETTE, D.; SYLVESTRE, S. (2002b). PAHs in Fraser River Basin: a critical appraisal of PAH ratios as indicators of PAH source and composition. Org. Geochem. 33: 429 - 515.

ZANARDI, E. BÍCEGO, M. C. MIRANDA, L. B. WEBER, R. R. (1999). Distribuition and Origin of Hydrocarbons in Water and Sediment in São sebastião, SP, Brazil. Marine Pollution Bulletim. N $\square$ 4, V. 38, 261-267.

ZHOU, J.L., HONG, H., ZHANG, Z., MASKAOUI, K., CHEN, W., (2000). Multiphase distribution of organic micropollutants in Xiamen harbor, China. Water Res. 34, 21322150 . 
Tabela 1: Concentrações (ng. $\mathrm{g}^{-1}$, peso seco) do total dos 24 HPAs e de Carbono orgânico (mg/g, peso seco) analisados nas amostras de sedimentos superficiais coletados na BTS.

\begin{tabular}{|c|c|c|c|c|c|c|c|c|c|c|c|c|c|c|c|c|c|c|}
\hline Estações & M1 & M2 & M3 & M4 & M5 & M6 & M7 & M8 & M9 & M10 & M11 & M12 & M13 & M14 & M15 & M16 & M17 & M18 \\
\hline Corg $(\mathrm{mg} / \mathrm{g})$ & 4,16 & 5,69 & 7,36 & 1,73 & 1,77 & 1,82 & 2,34 & 3,04 & 7,44 & 2,74 & 20,33 & 5,64 & 12,80 & 10,22 & 12,54 & 5,41 & 7,39 & 7,73 \\
\hline$\sum$ HPAs & 45,5 & 45,5 & 7,6 & 17,3 & 15,4 & 6,6 & 2,0 & 9,8 & 4,1 & 19,9 & 9,4 & 391,1 & 320,4 & 988,4 & 89,0 & 46,8 & 91,5 & 22,9 \\
\hline \multicolumn{19}{|l|}{ 2-3 anéis $(\mathrm{ng} / \mathrm{g})$} \\
\hline$\Sigma$ HPAs & 162,0 & 204,7 & 287,7 & 55,1 & 45,3 & 86,6 & 46,6 & 461,0 & 208,1 & 605,0 & 190,7 & 4573,6 & 2985,7 & 6232,9 & 2535,1 & 1060,5 & 6024,6 & 350,6 \\
\hline \multicolumn{19}{|l|}{ 4-6 anéis $(\mathrm{ng} / \mathrm{g})$} \\
\hline TotalHPAs(ng/g) & 207,5 & 250,2 & 295,3 & 72,4 & 60,7 & 93,2 & 48,6 & 470,8 & 212,2 & 624,9 & 200,1 & 4964,7 & 3306,1 & 7221,3 & 2624,1 & 1107,3 & 6116,0 & 373,5 \\
\hline
\end{tabular}


SANT'ANNA Jr. et al. Hidrocarbonetos policíclicos aromáticos em sedimentos superficiais na Baía de Todos os Santos - Nordeste do Brasil.

Tabela 2: Comparação do $\square$ HPAs ( $\mathrm{ng} / \mathrm{g}$ ) encontrado em amostras de sedimentos superficiais em várias partes do Mundo.

\begin{tabular}{|c|c|c|c|c|}
\hline Local & $\begin{array}{l}\text { Total de HPAs } \\
\text { Analisados }\end{array}$ & $\begin{array}{l}\text { इHPAs } \\
\left.\text { (ng.g }^{-1}\right) \\
\text { (Lim. Sup. - } \\
\text { Lim. Inf.) }\end{array}$ & $\begin{array}{l}\text { Nível de } \\
\text { Contaminação }\end{array}$ & Referência \\
\hline $\begin{array}{l}\text { BTS - Bahia / } \\
\text { Brasil }\end{array}$ & 24 & $0,3-7221,3$ & Baixo - Alto & Este Trabalho \\
\hline $\begin{array}{l}\text { Santos - SP / } \\
\text { Brasil }\end{array}$ & & $80-42.390$ & Baixo - Alto & $\begin{array}{l}\text { Nishigima et al. } \\
2001\end{array}$ \\
\hline $\begin{array}{l}\text { São Sebastião - } \\
\text { SP/ Brasil }\end{array}$ & 24 & $1,9-254,3$ & $\begin{array}{l}\text { Baixo - } \\
\text { Moderado }\end{array}$ & $\begin{array}{l}\text { Zanardi et al. } \\
1999\end{array}$ \\
\hline Caribe & & $103-1.656$ & $\begin{array}{l}\text { Baixo - } \\
\text { Moderado }\end{array}$ & $\begin{array}{l}\text { Bernanrd et al., } \\
1996\end{array}$ \\
\hline França & 17 & $45,8-4.888$ & Baixo - Alto & $\begin{array}{l}\text { Budzinsk et al., } \\
1997\end{array}$ \\
\hline Itália & 14 & $\begin{array}{l}86,5- \\
48.090\end{array}$ & Baixo - Alto & $\begin{array}{l}\text { Benlahcen et al., } \\
1997\end{array}$ \\
\hline França/Espanha & 14 & $1,2-8.420$ & Baixo - Alto & $\begin{array}{l}\text { Baumard et al., } \\
\text { 1998a }\end{array}$ \\
\hline $\begin{array}{l}\text { Baía de Arcachon / } \\
\text { França }\end{array}$ & 16 & $31,7-4.120$ & Baixo - Alto & $\begin{array}{l}\text { Baumard et al., } \\
\text { 1998b }\end{array}$ \\
\hline Mar Báltico & 14 & $3-30.100$ & Baixo - Alto & $\begin{array}{l}\text { Baumard et al., } \\
1999\end{array}$ \\
\hline China & 16 & $247-480$ & Moderado & $\begin{array}{l}\text { Zhou et al., } \\
2000\end{array}$ \\
\hline França & 14 & $3,5-853$ & $\begin{array}{l}\text { Baixo - } \\
\text { Moderado }\end{array}$ & $\begin{array}{l}\text { Soclo et al., } \\
2000\end{array}$ \\
\hline Nigéria & 14 & $25,1-1.411$ & $\begin{array}{l}\text { Baixo - } \\
\text { Moderado }\end{array}$ & $\begin{array}{l}\text { Soclo et al., } \\
2000\end{array}$ \\
\hline Hong Kong & & $4,2-11.098$ & Baixo - Alto & Tam et al., 2001 \\
\hline Itália & 22 & $\begin{array}{l}34,67- \\
682,3\end{array}$ & $\begin{array}{l}\text { Baixo - } \\
\text { Moderado }\end{array}$ & Notar et al. 2001 \\
\hline México & 16 & $7,6-813,1$ & $\begin{array}{l}\text { Baixo - } \\
\text { Moderado }\end{array}$ & $\begin{array}{l}\text { Macías-Zamora } \\
\text { et al., } 2002\end{array}$ \\
\hline China & 16 & $59-1.177$ & $\begin{array}{l}\text { Baixo - } \\
\text { Moderado }\end{array}$ & $\begin{array}{l}\text { Maskaoui et al., } \\
2002\end{array}$ \\
\hline China & 25 & $\begin{array}{l}97,6- \\
1.376,5\end{array}$ & $\begin{array}{l}\text { Baixo - } \\
\text { Moderado }\end{array}$ & Ou et al., 2004 \\
\hline China & 16 & $464-2.621$ & Moderado - Alto & Xu et al., 2007 \\
\hline Argentina & 18 & $\begin{array}{l}1,024- \\
10,261\end{array}$ & Baixo - Alto & $\begin{array}{l}\text { Arias et al., } \\
2010\end{array}$ \\
\hline
\end{tabular}


Tabela 3: Valores característicos de razões moleculares para distinguir fontes pirolíticas e petrogênicas.

\begin{tabular}{llll}
\hline Razão & Origem Pirolítica & $\begin{array}{l}\text { Origem } \\
\text { Petrogênica }\end{array}$ & Este Estudo \\
\hline Ant / Ant + Fen & $>0.10$ & $<0.10$ & $0.03-0.78$ \\
B(a)Ant/B(a)Ant+Cris & $>0.20$ & $<0.20$ & $0.13-0.41$ \\
Fluor / Fluor + Pir & $>0.40$ & $<0.40$ & $0.08-0.60$ \\
\hline
\end{tabular}

Tabela 4: Critérios de qualidade ambiental para 10 HPAs determinados pela agência ambiental do Canadá e concentração destes mesmos HPAs encontrados nas amostras coletadas na BTS e no litoral Norte da Bahia (Lee et al., 1999).

\begin{tabular}{lllcc}
\hline & & & \multicolumn{2}{c}{ Este Trabalho } \\
\cline { 4 - 5 } Composto & TEL & PEL & Média & $\begin{array}{c}\text { Lim. Inf. - Lim. } \\
\text { Sup. }\end{array}$ \\
\hline Naftaleno & 34,6 & 391 & 3,73 & $0,3-11,3$ \\
Acenaftileno & 5,87 & 128 & 0,59 & $0,2-1,5$ \\
Acenafteno & 6,71 & 88,9 & 5,91 & $0,2-44,6$ \\
Fluoreno* & 21,2 & 144 & 9,42 & $0,1-79,8$ \\
Fenantreno* & 86,7 & 544 & 70,0 & $0,1-668,8$ \\
Antraceno* & 46,9 & 245 & 17,0 & $0,1-152,1$ \\
Fluoranteno** & 113 & 1494 & 115,5 & $0,1-493,3$ \\
Pireno* & 153 & 1398 & 102,1 & $0,1-596,9$ \\
B(a)Antraceno** & 74,8 & 693 & 70,9 & $0,1-403,2$ \\
Criseno** & 108 & 846 & 196,1 & $0,3-1184,6$ \\
B(a)Pireno*** & 88,8 & 763 & 281,4 & $3,4-1647,1$ \\
\hline
\end{tabular}

